\title{
Evaluating the effectiveness of gloves in reducing the hazards of hand-transmitted vibration
}

\author{
Michael J Griffin
}

\begin{abstract}
Objectives-A method of evaluating the effectiveness of gloves in reducing the hazards of hand-transmitted vibration is proposed.

Method-The glove isolation effectiveness was calculated from: $(a)$ the measured transmissibility of a glove, $(b)$ the vibration spectrum on the handle of a specific tool (or class of tools), and (c) the frequency weighting indicating the degree to which different frequencies of vibration cause injury. With previously reported tool vibration spectra and glove transmissibilities (from 10-1000 Hz), the method was used to test 10 gloves with 20 different powered tools.
\end{abstract}

Results-The frequency weighting for hand-transmitted vibration advocated in British standard 6842 (1987) and international standard 5349 (1986) greatly influences the apparent isolation effectiveness of gloves. With the frequency weighting, the gloves had little effect on the transmission of vibration to the hand from most of the tools. Only for two or three tools (those dominated by high frequency vibration) did any glove provide useful attenuation. Without the frequency weighting, some gloves showed useful attenuation of the vibration on most powered tools.

Conclusions-In view of the uncertain effect of the vibration frequency in the causation of disorders from handtransmitted vibration, it is provisionally suggested that the wearing of a glove by the user of a particular vibratory tool could be encouraged if the glove reduces the transmission of vibration when it is evaluated without the frequency weighting and does not increase the vibration when it is evaluated with the frequency weighting. A current international standard for the measurement and evaluation of the vibration transmitted by gloves can classify a glove as an antivibration glove when it provides no useful attenuation of vibration, whereas a glove providing useful attenuation of vibration on a specific tool can fail the test.

(Occup Environ Med 1998;55:340-348)

Keywords: vibration; gloves; vibration-induced white finger; standards

Int Inturs of

University of Southampton,

Southampton SO17 1BJ

UK. Tel: 00441703592277 ;

Fax: 00441703 592927;

Email: mjg@isvr.soton.ac.uk

Accepted 31 October 1997

Occupational exposures to hand-transmitted vibration are associated with various vascular, neurological, and articular disorders. The pre- cise roles of the various physical characteristics of vibration (magnitude, frequency, direction, duration of exposure) required for the production of the disorders are not known. Even so, consensus standards suggest methods whereby the incidence of one of these disorders (vibration-induced white finger) can be predicted from measurements of vibration on tools. Measures taken to reduce vibration induced white finger are sometimes assumed to also minimise the development of other disorders. One preventive measure that might be considered is the wearing of gloves. Apart from any benefit in protecting the hands from abrasion, extremes of temperature, chemicals, and wetness, gloves might also be assumed to reduce the transmission of vibration to the hand.

In the past decade, as pressures to reduce occupational exposures to hand-transmitted vibration have increased, there has been increased commercial interest in the production of antivibration gloves. A recognition that some claims for the benefits of gloves were not well founded in engineering or medical knowledge led to the publication of international standard (ISO) 10819 in $1996 .{ }^{1}$ This standard defines a method for the measurement of glove dynamic performance and specifies the performance requirements for antivibration gloves.

The dynamic performance of a glove depends on the dynamic properties of materials either side of the glove (including the hand) and depends on the frequency of vibration. The effectiveness of a glove in reducing any vibration hazard will also depend on the characteristics of the vibration (especially the frequency spectrum) on a tool and the extent to which different vibration frequencies cause injury in the hand.

This paper critically reviews the form and content of ISO 10819. In view of fundamental deficiencies apparent in the standard, an alternative method based on the measurement of glove transmissibility and a consideration of the vibration spectra on individual tool types is investigated. The applicability of the standardised method and the alternative method are illustrated with previously reported measurements of glove transmissibility and tool vibration spectra.

\section{ISO 10819}

OUTLINE METHOD

The standard defines a procedure for deter- mining the extent to which two different vibration spectra (labelled $\mathrm{M}$ and $\mathrm{H}$ ) are transmitted through a glove to the hand. A standing subject 


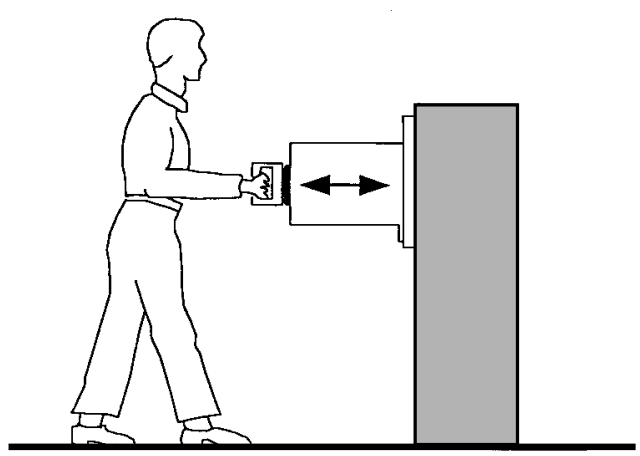

Figure 1 Configuration of subject and handle used to test the vibration transmissibility of gloves according to ISO 10819 (1996).

grips a vertically oriented cylindrical handle (40 $\mathrm{mm}$ diameter, $110 \mathrm{~mm}$ long) with a grip force of $30 \mathrm{~N} \pm 5 \mathrm{~N}$ and a push force of $50 \mathrm{~N} \pm$ $8 \mathrm{~N}$. The forearm is horizontal and parallel with the direction of the vibration while the wrist forms an angle between $0^{\circ}$ (neutral) and $40^{\circ}$ (dorsal bending) (fig 1).

Spectrum $M$ (medium frequencies) and spectrum $\mathrm{H}$ (high frequencies) are defined mathematically and in terms of their one third octave band acceleration values. Figure 2 shows both spectra before and after frequency weighting with the current frequency weighting for hand-transmitted vibration, called $\mathrm{W}_{\mathrm{h}}$ in British standard $6842 .^{23}$ Although the unweighted spectra are defined from 16 to $400 \mathrm{~Hz}$ (for $\mathrm{M}$ ) and from 100 to $1600 \mathrm{~Hz}$ (for $\mathrm{H}$ ), the
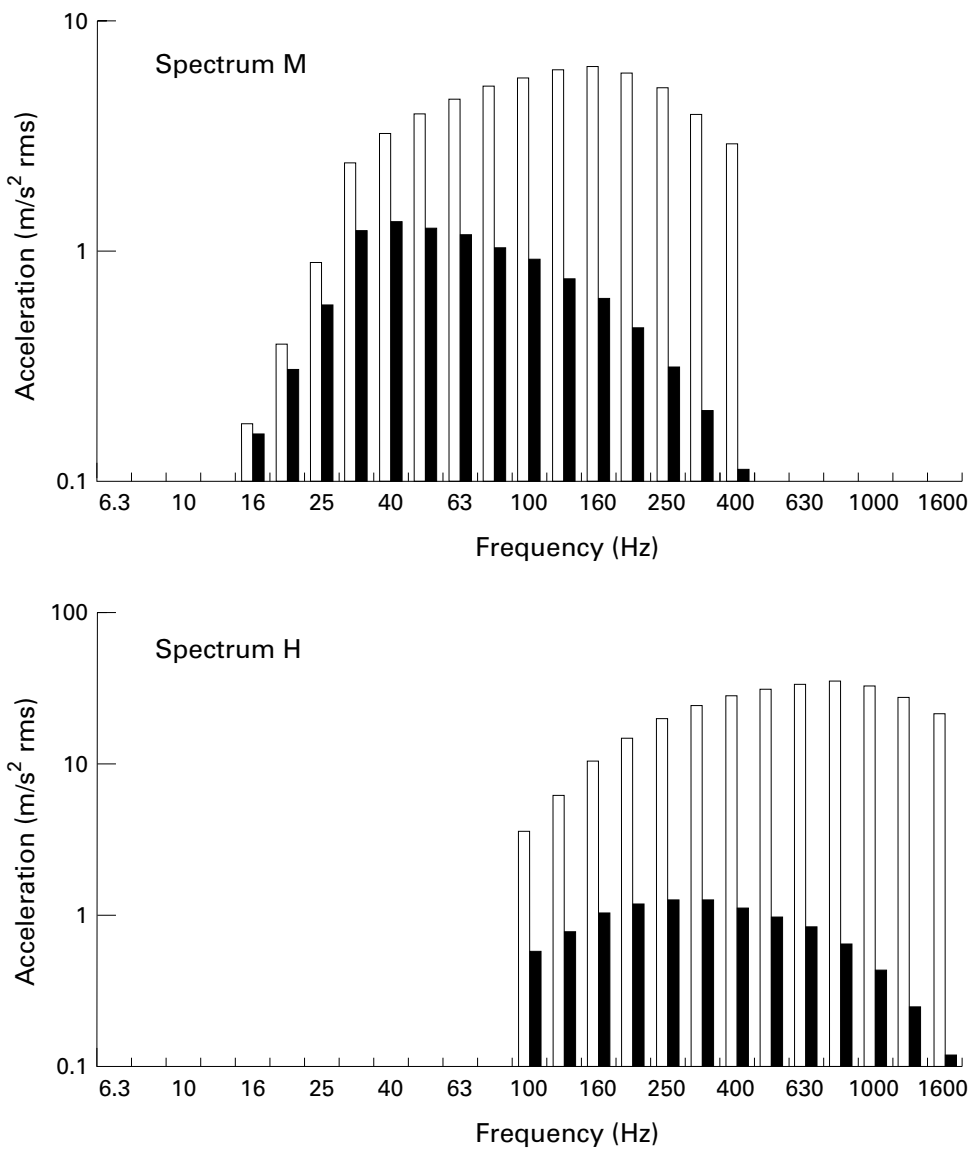

Figure 2 One third octave band spectra of spectrum $M$ and spectrum $H$ (open bars unweighted spectra; solid bars frequency-weighted spectra). significant energy in the weighted spectra may be considered to only extend from 25 to 200 $\mathrm{Hz}$ (for M) and from $100 \mathrm{~Hz}$ to $1000 \mathrm{~Hz}$ (for $\mathrm{H})$.

For both spectra, the so called transmissibility of a glove is determined by measuring the frequency-weighted acceleration on the handle and the frequency-weighted acceleration on a palm adapter held in the palm of the hand inside the glove. A drawing of this cusp shaped adapter (length of $70 \mathrm{~mm}$, width $31 \mathrm{~mm}$, thickness 7 to $15 \mathrm{~mm}$, and mass $\leqslant 15 \mathrm{~g}$ ) is given. The values are corrected for the transmissibility measured to the palm without the glove but, essentially, the transmissibility is given by the frequency-weighted acceleration on the palm adapter inside the glove divided by the frequency-weighted acceleration on the handle. The reported values for the two spectra (called the mean corrected transmissibility, $\overline{\mathrm{TR}}_{\mathrm{M}}$ and $\overline{\mathrm{TR}_{\mathrm{H}}}$ ) are the mean values of six measurements obtained with two repeats with each of three adult subjects with hand sizes in the range 7 to 9 according to European standard 420 .

The ISO $10819^{1}$ says:

"A glove shall not be considered as 'antivibration glove' according to this standard if it does not fulfil both of the following criteria:

$$
\overline{\mathrm{TR}}_{\mathrm{M}}<1.0 \text { and } \overline{\mathrm{TR}}_{\mathrm{H}}<0.6
$$

Note: the fulfilment of these criteria does not imply that the use of such gloves removes the hazard of vibration exposure."

\section{PROBLEMS}

Figure 3 compares spectra obtained from 20 different vibratory tools with the spectra $M$ and $\mathrm{H}$ defined in the standard. ${ }^{4}$ With the logarithmic scales in the figure it is clear that there are large differences between the measured spectra and spectra $\mathrm{M}$ and $\mathrm{H}$. Consequently, even if accurate and unbiased measurements could be made with the proposed method, the mean corrected transmissibilities $\left(\overline{\mathrm{TR}}_{\mathrm{M}}\right.$ and $\left.\overline{\mathrm{TR}}_{\mathrm{H}}\right)$ cannot be assumed to indicate whether a glove provides, or does not provide, useful attenuation of vibration when it is used with a specific tool. As tools do not usually have spectra similar to spectra $\mathrm{M}$ and $\mathrm{H}$, the performance of a glove when used with a tool should not be expected to be predictable from the values of $\mathrm{TR}_{\mathrm{M}}$ and $\mathrm{TR}_{\mathrm{H}}$ obtained with these spectra.

The procedure defined in the standard indicates the transmissibility to the palm of the hand: it does not indicate the extent to which a glove alters the transmission of vibration to the fingers, where vibration-induced injury is most often found. Hence, an antivibration glove could reduce the magnitude of vibration transmitted to the palm of the hand (according to the standard) but have no beneficial effect where the injury is found.

The standard says:

"Moreover, a glove shall only be considered as 'antivibration glove' according to this standard if the fingers of the glove have the same properties (materials and thickness) as the part of the glove covering the palm of the hand." 


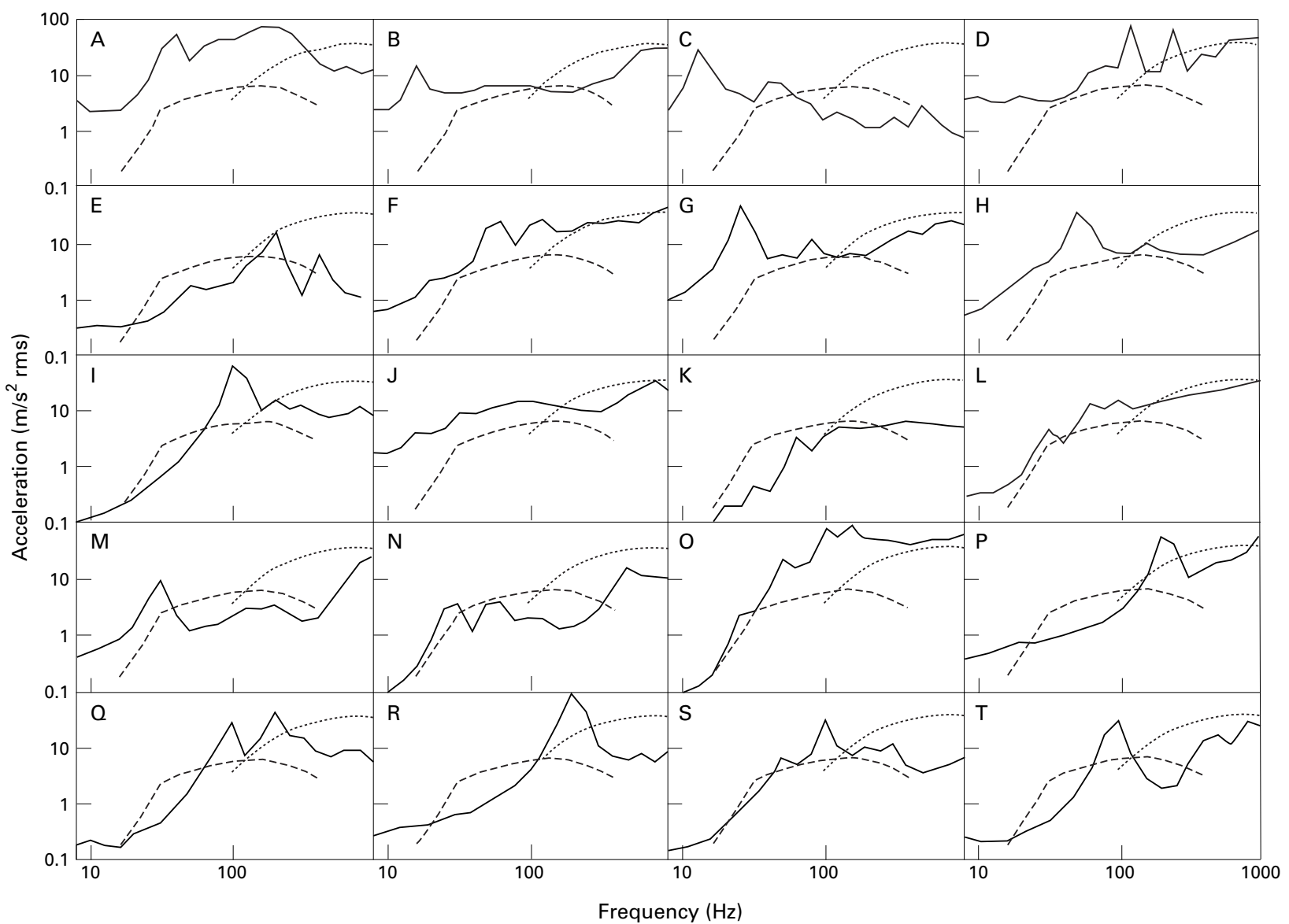

Figure 3 Comparison of spectrum $M(--)$ and spectrum $H(\ldots)$ with the spectra of 20 different powered hand tools $(\longrightarrow) .^{4}(A=$ pneumatic rock drill; $B=$ pneumatic road breaker; $C=$ petrol driven wacker compressing road surface after mending; $D=a$ non-antivibration chain saw; $E=$ an antivibration chain saw; $F=$ a pneumatic metal chipping hammer; $G=$ pole scabbler; $H=$ needle gun; $I=$ random orbital sander; $\mathcal{F}=$ impact wrench; $K=$ riveting gun; $L=$ dolly used with riveting gun; $M=$ nutrunner; $N=$ metal drill; $O=$ wire swaging; $P=$ etching pen; $Q=$ electric 9 inch angle grinder; $R=$ pneumatic rotary file; $S=$ pneumatic 5 inch straight grinder; $T=$ pneumatic 7 inch vertical grinder. All spectra from the axis giving the highest weighted acceleration.)

This clearly indicates the importance of glove material covering the fingers. However, this text is followed by a note:

"However, if such gloves fulfil the requirements regarding vibration transmissibility, they may have a beneficial effect when used in situations involving no contact between fingers and vibrating surface."

This apparently restricts the application of the entire standard to the use of gloves in situations where vibration is not transmitted through the material over the fingers.

The phrase "such gloves" is ambiguous in the note cited above: although grammatically it should refer to the gloves mentioned in the previous text (those with the same properties over the fingers) it might be taken to refer to gloves without suitable finger covering but with a $\overline{T R}_{\mathrm{M}}<1.0$ and $\overline{T R}_{H}<0.6$. With this interpretation, the standard would state that finger glove material is important and imply that antivibration gloves provide useful attenuation when there is finger contact.

According to the standard, the values of both $\overline{\mathrm{TR}}_{\mathrm{M}}$ and $\overline{\mathrm{TR}}_{\mathrm{H}}$ are to be based on the mean of six results (two measurements for each of three subjects). The SD and coefficient of variation are also required, although the basis for calculating these values is not specified. Some experience with the application of the method shows that the variation between tests with dif- ferent subjects can be greater than the variation between different gloves (discussed later). With only three subjects, the mean values obtained in one test may not be representative of those obtained in a repeated test with the same or different subjects in a later test. An indication of the required repeatability of measurements is necessary, and this may indicate the need for more than three subjects.

It is highly desirable that before a test procedure is promulgated as a standard it is tested in different laboratories so as to find the accuracy that can be achieved. It is not currently known with what accuracy different test laboratories will determine the values of $\overline{\mathrm{TR}}_{\mathrm{M}}$ and $\overline{\mathrm{TR}}_{\mathrm{H}}$ for the same gloves.

According to the standard, a glove can be described as an antivibration glove if the value of $\overline{\mathrm{TR}}_{M}$ is $<1.0$ and the value of $\overline{\mathrm{TR}}_{\mathrm{H}}$ is $<0.6$. In the case of $\overline{T R}_{M}$ the values will often be very close to 1.0. It is not clear what rounding of measurements is to be applied, but presumably a value of 0.99 would meet the criterion whereas a value of 1.0 would fail. However, the measurement accuracy and measurement repeatability - for example, subject variabilitywill not yield a precision of $1 \%$. A similar problem exists for $\mathrm{TR}_{\mathrm{H}}$. It would seem necessary to specify the assumed precision of measurements and it may be appropriate to combine a 


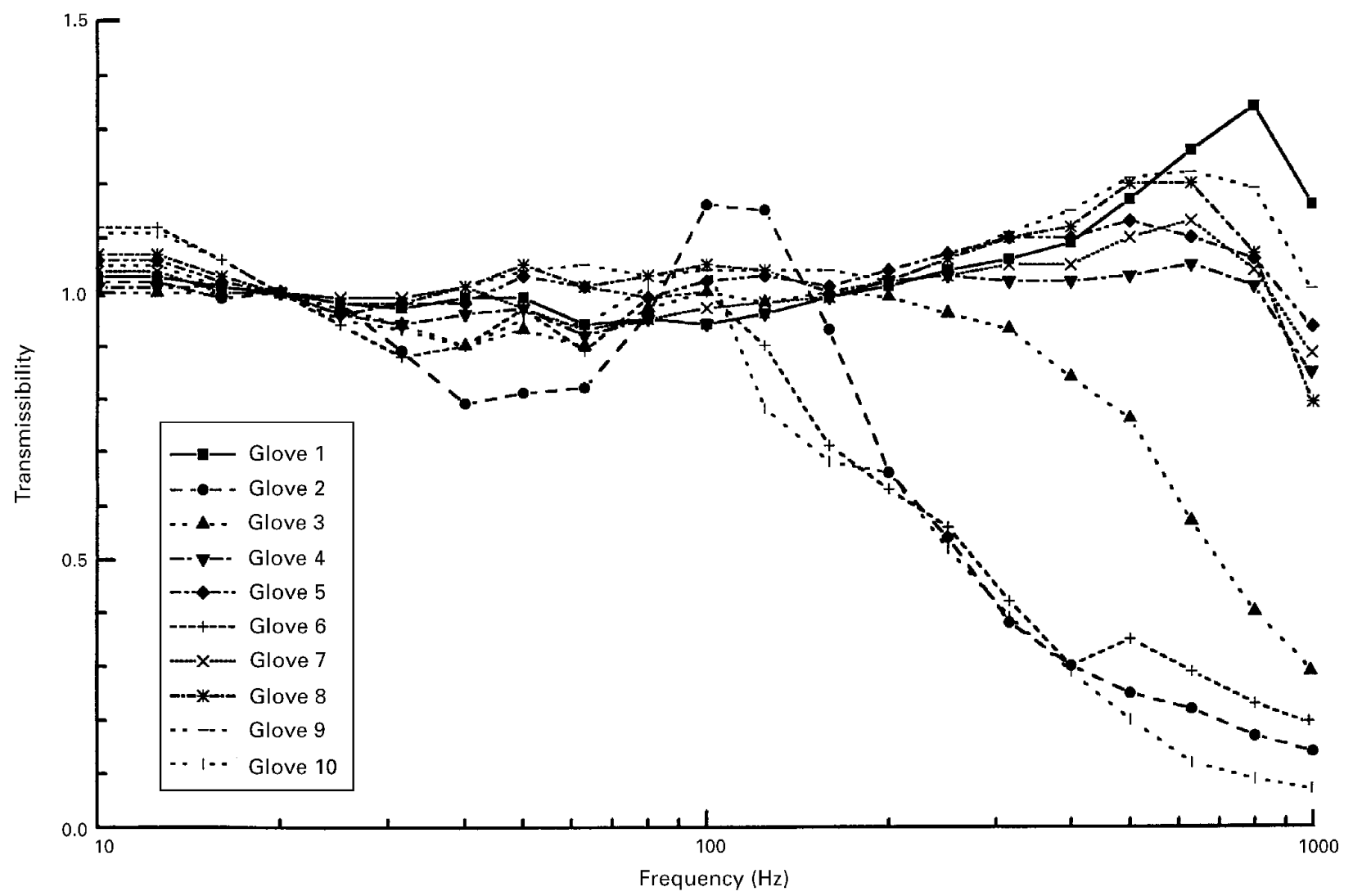

Figure 4 Transmissibilities of the 10 gloves measured from handle to palm adapter (values normalised to $1.0 \mathrm{at} 21 \mathrm{~Hz}$ ).

measure of the variability with the mean value before comparing the result with a criterion. For example, the quoted values could be $\left(\overline{T R}_{\mathrm{M}}+\delta_{\mathrm{M}}\right)$ and $\left(\overline{\mathrm{TR}}_{\mathrm{H}}+\left(\delta_{\mathrm{H}}\right)\right.$, where $\delta_{\mathrm{M}}$ and $\delta_{\mathrm{H}}$ are the SDs corresponding to the mean values of $\overline{\mathrm{TR}}_{\mathrm{M}}$ and $\overline{\mathrm{TR}}_{\mathrm{H}}$.

\section{Glove transmissibilities}

Figure 4 shows the median transmissibilities of 10 different gloves measured with eight subjects who used the palm adapter with a broad band spectrum of random acceleration (8$1260 \mathrm{~Hz}$ at $5 \mathrm{~ms}^{-2} \mathrm{rms}$, frequency-weighted). The transmissibilities obtained with individual subjects have been reproduced elsewhere, ${ }^{56}$ and show that the variability in transmissibility between these gloves at high frequencies is far greater than the variability between subjects, which is greater than the variability within a subject. The transmissibilities were normalised

Table 1 Comparison of measured and predicted values of $\overline{T R}_{M}$ and $\overline{T R}_{H}$ (Measured values from two repeats on each of three subjects; predicted values from median transmissibilities with eight subjects)

\begin{tabular}{rlllll}
\hline & \multicolumn{3}{l}{ Spectrum $M$} & & \multicolumn{2}{l}{ Spectrum $H$} \\
\cline { 2 - 3 } \cline { 5 - 6 } Glove & Predicted mean & $\begin{array}{l}\text { Measured } \\
\text { mean }(S D)\end{array}$ & & Predicted mean & $\begin{array}{l}\text { Measured } \\
\text { mean }(S D)\end{array}$ \\
\hline 1 & 0.97 & $0.98(0.05)$ & & 1.08 & $1.12(0.12)$ \\
2 & 0.90 & $1.14(0.18)$ & & 0.60 & $0.67(0.18)$ \\
3 & 0.94 & $1.11(0.11)$ & & 0.88 & $0.80(0.04)$ \\
4 & 0.95 & $1.07(0.11)$ & & 1.01 & $0.91(0.10)$ \\
5 & 1.0 & $1.09(0.15)$ & & 1.07 & $1.11(0.15)$ \\
6 & 0.91 & $1.08(0.15)$ & & 0.54 & $0.58(0.14)$ \\
7 & 0.98 & $1.07(0.14)$ & & 1.04 & $0.98(0.12)$ \\
8 & 1.02 & $1.09(0.17)$ & & 1.08 & $0.98(0.10)$ \\
9 & 1.03 & $1.11(0.18)$ & & 1.10 & $1.05(0.12)$ \\
10 & 0.92 & $1.16(0.18)$ & & 0.51 & $0.44(0.10)$ \\
\hline
\end{tabular}

to unity transmissibility at $21 \mathrm{~Hz}$ to compensate for any misalignment of the adapter with the direction of the vibration (this may not have fully compensated for the problem in this instance, as discussed later). Some gloves attenuated vibration at high frequencies (gloves 2,6 , and 10) whereas other gloves provided little or no attenuation at any frequency $<1000$ $\mathrm{Hz}$.

\section{Glove isolation effectiveness}

We use the term glove isolation effectiveness to indicate the extent to which a glove attenuates the effective vibration on a handle. The glove isolation effectiveness will depend on: $(a)$ the

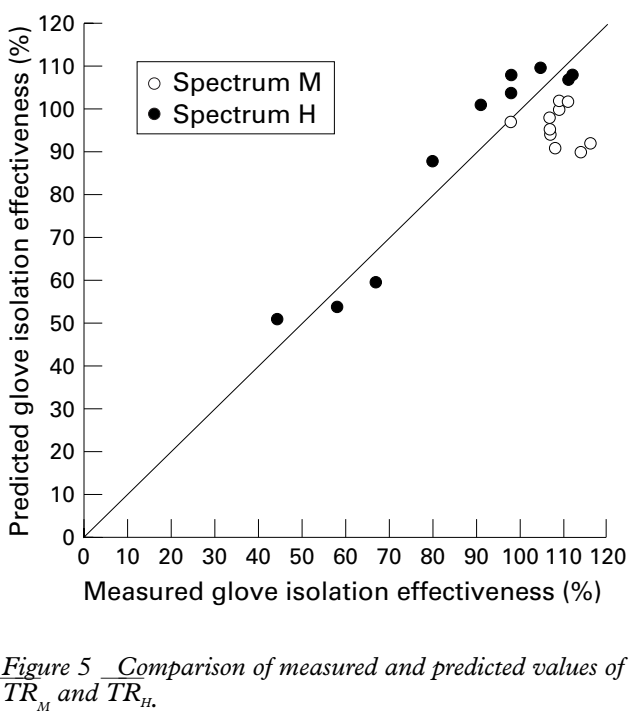


Table 2 Frequency-weighted measures of glove isolation effectiveness

\begin{tabular}{|c|c|c|c|c|c|c|c|c|c|c|}
\hline \multirow[b]{2}{*}{ Spectrum } & \multicolumn{10}{|c|}{ Glove } \\
\hline & 1 & 2 & 3 & 4 & 5 & 6 & 7 & 8 & 9 & 10 \\
\hline Spectrum $M$ & 97 & 90 & 94 & 95 & 100 & 91 & 98 & 102 & 103 & 92 \\
\hline Spectrum $H$ & 108 & 60 & 88 & 101 & 107 & 54 & 104 & 108 & 110 & 51 \\
\hline Pneumatic rock drill & 98 & 88 & 94 & 96 & 100 & 90 & 99 & 101 & 102 & 91 \\
\hline Pneumatic road breaker & 101 & 98 & 99 & 100 & 102 & 105 & 100 & 103 & 101 & 105 \\
\hline Petrol driven wacker & 103 & 101 & 100 & 101 & 105 & 111 & 103 & 106 & 104 & 110 \\
\hline Non-antivibration chain saw & 97 & 103 & 98 & 97 & 103 & 98 & 99 & 105 & 104 & 101 \\
\hline Antivibration chain saw & 98 & 96 & 98 & 98 & 102 & 85 & 99 & 102 & 103 & 84 \\
\hline Pneumatic metal chipping hammer & 97 & 89 & 93 & 95 & 102 & 91 & 97 & 103 & 104 & 92 \\
\hline Pole scabbler & 98 & 97 & 97 & 96 & 97 & 94 & 99 & 98 & 99 & 95 \\
\hline Needle gun & 98 & 83 & 93 & 96 & 102 & 95 & 97 & 104 & 104 & 95 \\
\hline Random orbital sander & 95 & 114 & 99 & 95 & 102 & 97 & 97 & 105 & 104 & 99 \\
\hline Impact wrench & 99 & 93 & 96 & 97 & 101 & 96 & 99 & 102 & 102 & 97 \\
\hline Riveting gun & 98 & 91 & 95 & 96 & 103 & 85 & 98 & 104 & 105 & 85 \\
\hline Dolly used with riveting gun & 98 & 89 & 94 & 95 & 101 & 88 & 97 & 103 & 104 & 90 \\
\hline Nutrunner & 98 & 91 & 95 & 95 & 98 & 91 & 99 & 99 & 100 & 94 \\
\hline Metal drill & 99 & 89 & 94 & 96 & 99 & 90 & 99 & 100 & 102 & 92 \\
\hline Wire swaging & 97 & 101 & 98 & 96 & 102 & 90 & 98 & 104 & 104 & 90 \\
\hline Etching pen & 103 & 66 & 96 & 101 & 104 & 64 & 102 & 103 & 104 & 65 \\
\hline Electric 9 inch angle grinder & 97 & 98 & 99 & 97 & 102 & 88 & 99 & 104 & 104 & 91 \\
\hline Pneumatic rotary file & 101 & 68 & 99 & 102 & 104 & 64 & 102 & 102 & 103 & 66 \\
\hline Pneumatic 5 inch straight grinder & 95 & 107 & 98 & 95 & 102 & 97 & 97 & 104 & 104 & 100 \\
\hline Pneumatic 7 inch vertical grinder & 95 & 107 & 98 & 95 & 101 & 98 & 97 & 104 & 104 & 101 \\
\hline
\end{tabular}

Values are percentages.

spectrum of vibration on the handle; $(b)$ the transmissibility of the glove (expressed as a function of frequency); $(C)$ the frequency weighting.

Glove isolation effectiveness is equivalent to seat effective amplitude transmissibility (SEAT values) used to assess the vibration isolation of seats. ${ }^{78}$ The values of $\overline{T R}_{\mathrm{M}}$ and $\overline{\mathrm{TR}}_{\mathrm{H}}$ in ISO 10819 (1996) give the glove isolation effectiveness with spectrum $M$ and spectrum $\mathrm{H}$ and the currently standardised frequency weighting, $\mathrm{W}_{\mathrm{h}}$.

It is possible to predict the values of $\overline{\mathrm{TR}_{\mathrm{M}}}$ and $\overline{\mathrm{TR}}_{\mathrm{H}}$ from the transmissibilities of the 10 gloves (fig 4). Table 1 compares the measured and predicted values of $\overline{\mathrm{TR}}_{\mathrm{M}}$ and $\overline{\mathrm{TR}}_{\mathrm{H}}$, and figure 5 shows the correlations between the measured and predicted values. Although the predicted values are calculated from the median transmissibilities obtained with eight subjects (fig 4 ), the measured values are the average values measured according to the standard with two repeats from each of three subjects. The large scatter of values for the different gloves with spectrum $\mathrm{H}$ gives a high correlation between measured and predicted values, but the small range of values with spectrum $M$ leaves no correlation between measured and predicted results. This suggests that with spectrum $M$ the differences between gloves were smaller than errors in either the measured or the predicted values (errors in the alignment of the adapter with the direction of vibration, or errors introduced by subject variability).

The glove transmissibility can also be used to calculate the glove isolation effectiveness for each of the 10 gloves when used with each of the 20 tools. Figure 3 shows the spectra of these:

$\begin{aligned} & \text { Glove isolation } \\ & \text { effectiveness } \%\end{aligned}=\left[\frac{\sum_{\mathrm{i}=1}^{\mathrm{i}=22} a_{i}^{2} H_{i}^{2} W_{i}^{2}}{\sum_{\mathrm{i}=1}^{\mathrm{i}=22} a_{i}^{2} W_{i}^{2}}\right]^{\frac{1}{2}} \times 100$

Table 3 Measures of glove isolation effectiveness obtained without a frequency weighting

\begin{tabular}{|c|c|c|c|c|c|c|c|c|c|c|}
\hline \multirow[b]{2}{*}{ Spectrum } & \multicolumn{10}{|c|}{ Glove } \\
\hline & 1 & 2 & 3 & 4 & 5 & 6 & 7 & 8 & 9 & 10 \\
\hline Spectrum $M$ & 99 & 88 & 96 & 98 & 103 & 81 & 99 & 104 & 104 & 80 \\
\hline Spectrum $H$ & 118 & 32 & 68 & 100 & 107 & 35 & 104 & 108 & 114 & 28 \\
\hline Pneumatic rock drill & 100 & 85 & 96 & 99 & 103 & 77 & 100 & 103 & 104 & 76 \\
\hline Pneumatic road breaker & 120 & 43 & 60 & 97 & 103 & 46 & 102 & 104 & 112 & 42 \\
\hline Petrol driven wacker & 102 & 99 & 99 & 100 & 105 & 108 & 103 & 106 & 104 & 107 \\
\hline Non-antivibration chain saw & 108 & 79 & 88 & 99 & 105 & 71 & 103 & 108 & 110 & 73 \\
\hline Antivibration chain saw & 99 & 92 & 98 & 99 & 102 & 73 & 100 & 102 & 105 & 70 \\
\hline Pneumatic metal chipping hammer & 112 & 59 & 74 & 97 & 103 & 56 & 100 & 103 & 109 & 53 \\
\hline Pole scabbler & 109 & 74 & 83 & 97 & 101 & 73 & 101 & 102 & 106 & 73 \\
\hline Needle gun & 104 & 74 & 85 & 96 & 102 & 82 & 97 & 103 & 106 & 81 \\
\hline Random orbital sander & 97 & 108 & 97 & 96 & 103 & 92 & 98 & 105 & 105 & 92 \\
\hline Impact wrench & 117 & 56 & 70 & 98 & 104 & 55 & 102 & 106 & 112 & 52 \\
\hline Riveting gun & 111 & 58 & 82 & 100 & 106 & 54 & 103 & 108 & 111 & 50 \\
\hline Dolly used with riveting gun & 115 & 48 & 70 & 98 & 104 & 47 & 101 & 105 & 111 & 44 \\
\hline Nutrunner & 120 & 35 & 48 & 92 & 99 & 37 & 96 & 94 & 108 & 33 \\
\hline Metal drill & 119 & 35 & 66 & 100 & 107 & 39 & 105 & 111 & 116 & 32 \\
\hline Wire swaging & 106 & 78 & 87 & 98 & 104 & 67 & 101 & 104 & 108 & 66 \\
\hline Etching pen & 111 & 46 & 75 & 97 & 102 & 46 & 99 & 99 & 106 & 44 \\
\hline Electric 9 inch angle grinder & 102 & 79 & 96 & 100 & 104 & 71 & 101 & 104 & 105 & 73 \\
\hline Pneumatic rotary file & 102 & 66 & 98 & 102 & 104 & 62 & 102 & 103 & 103 & 64 \\
\hline Pneumatic 5 inch straight grinder & 98 & 100 & 96 & 96 & 103 & 88 & 99 & 105 & 105 & 90 \\
\hline Pneumatic 7 inch vertical grinder & 113 & 71 & 74 & 96 & 103 & 65 & 100 & 104 & 110 & 65 \\
\hline
\end{tabular}

Values are percentages. 
Table 4 Some problems with the method specified in ISO 10819 (1996)

\begin{tabular}{|c|c|}
\hline General & $\begin{array}{l}\text { The vibration spectra on tools vary greatly, so the attenuation provided to only two spectra cannot indicate } \\
\text { the attenuation provided for all tools. } \\
\text { The spectra } M \text { and } \mathrm{H} \text { used in the standard are unlike the spectra of common vibratory tools. } \\
\text { It is possible to fail the test with a glove that provides useful attenuation of vibration on a particular tool. } \\
\text { It is possible to pass the test with a glove that provides no useful attenuation of the vibration on a particular } \\
\text { tool. } \\
\text { Subject variability may influence measurements such that a glove could be classed as an "antivibration } \\
\text { glove" with one group of three test subjects but fail the test with another group of three test subjects. } \\
\text { The repeatability of the test when performed in different test houses is not known. } \\
\text { The variability of measurements is not considered when judging whether } \mathrm{TR}_{M}<1.0 \text { and } \overline{T R}_{\mathrm{H}}<0.6 \text {. } \\
\text { The standard is ambiguous in stating that the fingers of an "antivibration glove" must have the same } \\
\text { material properties (materials and thickness) as the part of the glove over the palm: it is not clear whether } \\
\text { an "antivibration glove" can be assumed to provide benefit at the fingers. }\end{array}$ \\
\hline Method & $\begin{array}{l}\text { The method of correction using a bare hand may introduce errors; it will not significantly increase accuracy } \\
\text { if the method is correctly applied. } \\
\text { The orientation of the palm adapter within the glove is important but it is difficult to control and the } \\
\text { problem is not considered in the standard. } \\
\text { The accurate control of grip force complicates the method but does not seem necessary; controlling grip to } \\
\text { the specified force will not achieve the same grip pressure when different methods are used for measuring } \\
\text { grip force. }\end{array}$ \\
\hline Practical problems & $\begin{array}{l}\text { It is difficult to construct a handle without resonances which prevent conformance with the standard. Such } \\
\text { resonances could influence the measured values of } \overline{T R}_{M} \text { and } \overline{T R}_{H} \text { and there is no warning of the problem in } \\
\text { the standard. } \\
\text { The requirement to measure each vibration over a period of } 30 \text { seconds is an unnecessary burden. } \\
\text { Some tolerances seem unnecessarily restrictive (for example, for spectra at high frequencies, grip force, and } \\
\text { push force). }\end{array}$ \\
\hline Assumptions & $\begin{array}{l}\text { It is assumed that reported measures of } \overline{\mathrm{TR}}_{\mathrm{M}} \text { and } \overline{\mathrm{TR}}_{\mathrm{H}} \text { will be obtained with spectra which meet the } \\
\text { requirements of the standard: there is no check on the accuracy of achieving required tolerances on } \\
\text { spectrum } \mathrm{M} \text { and spectrum } \mathrm{H} \text {. } \\
\text { It is assumed that the palm adapter does not alter glove-hand dynamics. } \\
\text { It is assumed that the posture and orientation of the arm do not sufficiently change hand impedance and } \\
\text { alter the transmission of vibration to the palm of the hand. } \\
\text { It is assumed that the hazards of different frequencies of vibration are adequately reflected in the frequency } \\
\text { weighting } \mathrm{W}_{\mathrm{h}} \text {. }\end{array}$ \\
\hline
\end{tabular}

where $a_{i}, H_{i}$, and $W_{i}$ are the values of acceleration on the handle of a tool, median glove transmissibility (from eight subjects), and the frequency weighting $\left(\mathrm{W}_{\mathrm{h}}\right)$, respectively, for the 22 one third octave bands from 8 to $1000 \mathrm{~Hz}$.

Table 2 shows the predicted values of glove isolation effectiveness for the 10 gloves when exposed to spectra $\mathrm{M}$ and $\mathrm{H}$ and all 20 spectra from the 20 tools.

For the tools, significant useful attenuation of vibration - that is, a glove isolation effectiveness much less than $100 \%$-is only seen with three gloves used with two tools. For the high frequency vibration of the etching pen and the pneumatic rotary file, gloves 2, 6, and 10 gave values of glove isolation effectiveness in the range $64 \%-68 \%$. For all other combinations of tools and gloves, the glove isolation effectiveness varied around $100 \%(83 \%-114 \%)$. This provides little encouragement for the use of any glove to attenuate vibration unless the vibration spectrum is dominated by high frequency components.

The predicted values with spectrum $M$ and spectrum $\mathrm{H}$ may be compared with the range of values obtained for specific tools. With spectrum $M$, the values fall in a narrow range from $90 \%$ to $103 \%$, compared with a wider range of values for individual tools from $64 \%$ to $114 \%$. With spectrum $\mathrm{H}$, four gloves give values lower than achieved with the spectrum for any tool, and five gloves give values higher than achieved with any tool. It is therefore difficult to see any value in the results obtained with spectrum $M$ or spectrum $\mathrm{H}$.

The standardised method assumes that the harmful effects of hand-transmitted vibration are adequately predicted with the frequency weighting defined in ISO $5349,{ }^{3}$ British stand- ard $6842^{2}$ and other similar national standards. However, the frequency weighting is not proved to reflect the hazard caused by different vibration frequencies and some researchers have been unable to find any improvement in the prediction of injury from different exposure conditions by use of the frequency weighting. Table 3 therefore shows the predicted glove isolation effectiveness for each glove and tool combination without the use of the frequency weighting $\mathrm{W}_{\mathrm{h}}$.

Without the frequency weighting, the vibration on many tools is usefully attenuated by three gloves, 2, 6, and 10, with attenuation of the vibration on several tools also provided by glove 3. On the basis of the unweighted data, gloves 2,6 , and 10 could be advocated for use on all tools except the petrol driven wacker, the random orbital sander and, perhaps, the pneumatic 5 inch straight grinder. These gloves show several cases in which the unweighted acceleration predicted at the palm adapter is halved by the wearing of a glove.

Without the frequency weighting, spectrum $M$ seems to provide no overall indication of glove performance, whereas spectrum $\mathrm{H}$ again tends to either overestimate attenuation or overestimate amplification, depending on the glove.

\section{Discussion}

Table 4 lists some of the principal problems with ISO 10819. ${ }^{1}$ Technical problems with the standard are summarised in the appendix.

Glove isolation effectiveness is directly equivalent to seat effective amplitude transmissibility (SEAT values ${ }^{7}$ ). The SEAT value was introduced to allow a method of optimising seat dynamics to specific vehicles, but has 
subsequently been extended to groups of vehicles in various international standards ${ }^{9} 10$ and equivalent European standards and national standards. In these standards it is assumed that a vehicle of a particular type and weight is characterised by a specific spectrum of vertical vibration. The spectrum is defined in the standard and reproduced in laboratory tests: the process has been mirrored in the glove testing standard ISO 10819. However, the spectra $\mathrm{M}$ and $\mathrm{H}$ are not based on the spectra from any specific tools, they are arbitrary bands of frequency, possibly intended to show the extent to which a glove attenuates medium and high frequency vibration.

One way forward would be to restrict the method to specific tools. By defining spectra typical of tool groups it would be possible to see the benefits (or otherwise) of using a glove with a specific type of tool. The spectra might initially be restricted to those tools known to commonly cause vibration-induced injuries and might include tools in which the vibration can and cannot be attenuated, so as to inhibit the development of gloves tested for one type of tool but used with an inappropriate tool type. Any classification of an antivibration glove must be associated with its use with a particular class of tool.

It may not be necessary to reproduce many different types of vibration in a laboratory test to measure the glove isolation effectiveness for specific tool spectra. If the standard were to define a satisfactory method of measuring the transmissibility of the glove over a wide frequency range, the glove isolation effectiveness could be calculated for various classes of tool with the same methods used to predict the values in tables 2 and 3 of this paper. This would allow values to be calculated for new spectra introduced in later standards without retesting a glove. It will also encourage the prediction of glove isolation effectiveness for tools not included in any standard. (This type of prediction is not always appropriate with the SEAT values of suspension seats due to non-linearities, but it may be possible for gloves to an accuracy no less than that in the current ISO 10819.)

Measuring the glove transmissibility over a wide frequency range and restricting the measure of glove isolation effectiveness to the spectra from specific tools may partially overcome doubts with the frequency weighting, $\mathrm{W}_{\mathrm{h}}$. For a tool with a single dominant frequency (such as the pneumatic rotary file) the same measure of effectiveness will be obtained with or without the frequency weighting. With tools with somewhat broader vibration spectra it can be argued whether either a weighted or an unweighted measure of glove isolation effectiveness will give a better indication of glove performance. The weighted values will often suggest less attenuation than the unweighted values. Perhaps the standard should require that both values should be presented, with the recommendation that no glove should be sold for use with a vibratory tool if either value exceeds 1.0. If research leads to modifications in the frequency weighting for hand- transmitted vibration, this provisional recommendation should be reviewed. The current advantage is that whereas the weighting $\mathrm{W}_{\mathrm{h}}$ attaches great importance to low frequency acceleration, the evaluation without a frequency weighting gives more importance to higher frequencies. Neither approach will be optimum for an injury mostly caused by intermediate frequencies and not produced by either low or high frequency vibration. This is likely to be the case for some disorders caused by hand-transmitted vibration, with different injuries having different intermediate frequencies at which injuries are more likely. On this basis, it can be foreseen that a subsequent standard might require the presentation of measures of glove isolation efficiency calculated with more than one frequency weighting.

The transmissibility of a glove shows the response of the dynamic interaction between the glove and the hand: it is a "signature" of the hand-glove system. As such it must have a recognisable form with near unity transmissibility at low frequency, and probably some evidence of resonance before providing attenuation at high frequencies. The form of a glove transmissibility is therefore evidence of its validity as well as providing information which can be used to decide on the usefulness of the glove in attenuating specific vibration spectra. In contrast, the values of $\overline{\mathrm{TR}}_{\mathrm{M}}$ and $\overline{\mathrm{TR}}_{\mathrm{H}}$ do not signify any particular dynamic response: they give no indication as to the validity of the measurements (or predictions) on which they are based and cannot be used to assess the isolation to be provided for any spectra other than spectrum $M$ and spectrum $\mathrm{H}$.

Experience in at least two laboratories has shown that it can be difficult to maintain the orientation of the palm adapter within the glove and that this can alter the values of $\overline{T R}_{M}$ and $\overline{\mathrm{TR}}_{\mathrm{H}}{ }^{5}{ }^{11}$ The presentation of transmissibilities as a function of frequency could provide one solution to this problem: on the assumption that the transmissibility is unity at a low frequency, small deviations from unity might be assumed to be caused by malalignment and corrected by normalisation, as in figure 4 . However, other solutions to this problem are possible (see appendix).

\section{Conclusion}

The ISO 10819 does not provide a convenient or sufficient means of evaluating the vibration isolation performance of gloves. The standard can classify a glove as an antivibration glove when it provides no useful attenuation of vibration on the handle of a tool, whereas a glove which does provide useful attenuation of vibration on a specific tool can fail the test. A test based on the response of gloves to two spectra-for example, spectrum $M$ and spectrum $\mathrm{H}$-cannot indicate the overall effectiveness of gloves for any vibratory tool. The response to spectrum $M$ is likely to be close to unity whereas the response to spectrum $\mathrm{H}$ can suggest greater attenuation than will be realised when using the glove on most common vibratory tools. Any specification of an antivibration glove should be associated with the 
characteristics of a specific vibration spectrum; the recommended use of such gloves should be restricted to vibratory tools having spectra similar to those for which the vibration properties of the glove were evaluated.

With the frequency weighting advocated in all current standards, the results presented in table 2 suggest that for most vibratory tools the attenuation or amplification provided by the gloves was minimal, or within the range of measurement error. Only for two or three tools dominated by high frequency vibration was some attenuation provided, and this was only shown by three gloves constructed from the more compliant materials. Without the frequency weighting (table 3), many of the gloves still provided no useful attenuation of the vibration on any vibratory tool, but three of the gloves gave useful attenuation of the vibration on most tools. In view of doubts as to the validity of the frequency weighting, it is provisionally suggested that the wearing of a glove may be advisable if the glove: (a) does not increase the overall magnitude of vibration when evaluated with the frequency weighting, and (b) decreases the overall magnitude of vibration when evaluated without the frequency weighting (over a specified bandwidth-for example, 8-1000 Hz).

\section{Appendix: Technical problems with ISO 10819 (1986)}

HANDLE RESONANCES

The specified equipment requires a handle capable of indicating grip force over the range $10-50 \mathrm{~N}$ (with a resolution of $2 \mathrm{~N}$ ) yet which can reproduce the vibration spectra to an accuracy of $1 \mathrm{~dB}$ over the range $31.5-1000 \mathrm{~Hz}$ and to within $\pm 2 \mathrm{~dB}$ at frequencies down to $16 \mathrm{~Hz}$ and up to $1500 \mathrm{~Hz}$. The standard contains a figure giving an example design of a handle, but it is doubtful whether a handle of the type illustrated would be sufficiently rigid over the required range of frequencies. The standard does not require evidence that the vibration spectra were reproduced accurately and so it is not known whether handles currently used have resonances at frequencies sufficiently above $1500 \mathrm{~Hz}$ to allow the tolerance specified in the standard to be met. A resonance would tend to increase the proportion of high frequency vibration on the handle, which would be attenuated by some gloves, and so give rise to artificially low values of $\overline{\mathrm{TR}}_{\mathrm{H}}$. So long as the current frequency weighting $\left(\mathrm{W}_{\mathrm{h}}\right)$ is used, the high frequency spectrum could be limited to a frequency no higher than about $1000 \mathrm{~Hz}$ without loss of accuracy. For the current method to be more practical and robust, the tolerances for the spectra should be more reasonable and measurements of $\overline{\mathrm{TR}}_{\mathrm{M}}$ and $\overline{\mathrm{TR}}_{\mathrm{H}}$ should be accompanied by evidence that the spectra have been reproduced within the required tolerances.

CORRECTION METHOD

The standard requires that the weighted acceleration has a magnitude of $3.4 \mathrm{~ms}^{-2} \mathrm{rms}$ for spectrum $M$ and $3.3 \mathrm{~ms}^{-2} \mathrm{rms}$ for spectrum $\mathrm{H}$, both with a tolerance of $\pm 10 \%$. It also requires that the unweighted acceleration measured in the palm adapter held on the handle with a bare hand is within $5 \%$ of the unweighted acceleration measured on the handle. The weighted acceleration on the palm adapter within the glove is then corrected by the weighted acceleration measured with the bare hand. The correction is presumably intended to correct for systematic errors $<5 \%$. It seems optimistic to seek an accuracy $>5 \%$, and even if $5 \%$ is achieved it will not show that the adapter is receiving the same spectrum as that on the handle: resonances of the handle and rocking motions of the adapter could alter the motions measured in the palm adapter resting on the handle unlike the way they are altered when the adapter is worn within a glove. The use of this measurement to correct other measurements seems either insufficient or unnecessary, and it is a potential source of error.

The standard suggests that if the phase measured between acceleration on the handle and acceleration in the palm adapter with the bare hand is greater than \pm 5 degrees, the measurement should be considered not valid. However, the method of measuring phase is not specified, the measurement of phase is not mandatory and it is doubtful whether this tolerance can often be achieved with spectra $M$ and $\mathrm{H}$ over the full frequency ranges for which they are specified.

GRIP MEASUREMENT

Problems with resonances in the handle would be eased by eliminating the requirement to measure grip force in the handle. With the adapter in place within some gloves it is difficult for subjects to place their fingers around the specified handle to apply the required grip. The standard requires grip force to be measured to a very high accuracy, yet different methods of measuring the grip force are likely to result in forces within the palm of the hand which differ by values greater than the required grip measurement accuracy. Push force may be expected to alter the compression of the glove material in the palm of the hand and can be measured without instrumenting the handle, whereas grip force requires instrumentation likely to reduce the resonance frequency of the handle. Some studies have shown that grip force seems to have little effect on glove dynamic properties when the push force is controlled. ${ }^{5}$ It may therefore be better to eliminate the control of grip force when using the current method.

\section{POSTURE, ORIENTATION OF THE ARM, AND} DIRECTION OF VIBRATION

The transmissibility of a glove depends on the mechanical impedance of the hand and arm. The tests are conducted with a specific orientation of the body which will result in a specific hand-arm impedance. Although this may aid comparison between measurements in a simple test, it may provide an inaccurate indication of the isolation effectiveness of gloves used with different arm postures. The standard is also limited to vibration occurring 
normal to the palm of the hand, which is not always the direction of vibration with the highest magnitude. The standard should be extended to include other directions of vibration and consider other postures.

\section{ORIENTATION OF THE ADAPTER}

When the adapter is placed within a glove it is not visible to the experimenter who is unable to check whether it is aligned with the axis of vibration. Any alignment error will reduce the value measured within the glove and give rise to artificially low values of $\overline{\mathrm{TR}}_{\mathrm{M}}$ and $\overline{\mathrm{TR}}_{\mathrm{H}}$. Small errors may not have a large effect as, in theory, the reduction decreases in proportion to the cosine of the alignment error. However, experience has shown that errors as large as 30 degrees can easily occur, reducing the measured transmissibility by $\geqslant 15 \%$. There are several alternative solutions to this problem: the palm adapter could contain a small and suitably oriented accelerometer with a response to gravity acting as an inclinometer, or the glove material could be cut so as to make the adapter visible during the test.

\section{SUBJECT CHARACTERISTICS}

In recognition that the wearer of a glove may affect the transmissibility of a glove, the reported values are based on the results from three subjects. Although hand size is controlled, the measured glove transmissibility will depend on hand-arm impedance, which is not controlled. Some results show that the variability between transmissibilities measured with different subjects can be larger than the differences between many gloves, especially with spectrum $\mathrm{M}^{6}{ }^{6}$ It will therefore be possible to obtain artificially low or artificially high values by the selection of subjects. The standard would seem to allow testing to be performed with a large group of subjects and then the selection of the results from three subjects with the most favourable results.

EFFECT OF HAND ADAPTER

It seems probable that the hand adapter will sometimes alter the dynamic interaction between the hand and the glove by one or more of the following: influencing the impedance match between glove and hand ${ }^{12}$; influencing the glove dynamics (for example, preventing lateral stretching of compliant material $)^{13}$; influencing the hand dynamics (for example, by abnormal compression of hand tissues); changing the contact area with the source of vibration $^{14}$; or changing the radius of grip of the hand (with thick gloves, the additional depth of the adapter makes it difficult to grip the fingers around the specified handle).

The impedance effect of the adapter is not known but can be expected to be small at low frequencies and high at high frequencies. It may be expected to reduce the resonance frequency of the glove-hand combination and increase the attenuation at high frequencies. ${ }^{12}$

1 International Organization for Standardization. Mechanical vibration and shock-hand-arm vibration-method for the measurement and evaluation of the vibration transmissibility of gloves at the palm of the hand. Geneva: ISO, 1996:10819.

2 British Standards Institution. Measurement and evaluation of human exposure to vibration transmitted to the hand. London: human exposure to

3 International Organization for Standardization. Mechanical vibration - guidelines for the measurement and the assessment of human exposure to hand-transmitted vibration. Geneva: ISO, 986:5349

4 Griffin MJ. Measurement, evaluation and assessment of occupational exposures to hand-transmitted vibration. Occup Environ Med 1997;54:73-89.

5 Paddan GS. Effect of grip force and arm posture on the transmission of vibration through gloves. Proceedings of the United Kingdom Informal Group Meeting on Human Response to Vibration. Nuneaton, Warwickshire: Motor Industry Research Association, 18-20 September 1996.

6 Paddan GS, Griffin MJ. Individual variability in the transmission of vibration through gloves. Ergonomics rary Ergonomics 1997, Proceedings of the Annual Conference of the Ergonomics Society. London: Taylor Francis, 1997: $320-5$.

7 Griffin MJ. The evaluation of vehicle vibration and seats. Applied Ergonomics 1978;1:15-21.

8 Griffin MJ. Handbook of human vibration. London: Academic Press, 1990

9 International Organization for Standardization. Agricultural wheeled tractors - operator's seat-laboratory measurement of transmitted vibration. Geneva: ISO, 1990:5007.

10 International Organization for Standardization. Earthmoving machinery-laboratory evaluation of operator seat vibration. Geneva: ISO, 1994:7096.

11 Hewitt SM. Development of a test facility for anti-vibration gloves. Proceedings of the United Kingdom Informal Group Meeting on Human Response to Vibration. Nuneaton, 18-20 September 1996.

12 Griffin MJ, Macfarlane CR, Norman CD. The transmission of vibration to the hand and the influence of gloves. In: Brammer AJ, Taylor W, eds. Vibration effects on the hand and arm in industry. New York: John Wiley, 1982:103-16.

13 Mann NAJ. Measuring the vibration transmissibility through glove materials to the hand. Proceedings of the United Kingdom Informal Group Meeting on Human Response to Vibration, held at The Army Personnel Research Establishment, Ministry of Defence, Farnborough. Farnborough: Army Personnel Research Establishment, 20-22 September, 1993.

14 Mann NAJ, Griffin MJ. Effect of contact conditions on the mechanical impedance of the finger. Cent Eur $\mathcal{f}$ Public Health 1996;4. (JHEMI, 40). 